\title{
Remote Ecg Monitoring System by using Iot
}

\author{
Pagadala Pavan Kumar, Thokala Santhosh Naidu, S.Vishnu
}

\begin{abstract}
In this paper, we proposed a prototype to monitor the heartbeat of a patient perpetually through the cloud. The AD8232 sensor is utilized to quantify the heartbeat. Nodemcu ESP 8266 microcontroller is utilized to process the data like transmitting and receiving the data. The data from the heartbeat sensor is accumulated through the microcontroller and the data is posted into the cloud with the avail of Thingspeak. This model is very secure in terms of data because the target person is connected to the medico predicated on the unique IP address which provides us a secure transmission of data. This model consumes less power and withhalf the precision is more.
\end{abstract}

Keywords-Microcontroller; Thingspeak; (key words)

\section{INTRODUCTION}

Nowadays Non-communicable diseases are alarming at a higher rate in the world. According to the WHO Reports Non-communicable diseases like cardiovascular diseases(heart diseases coronary stroke and hypertension)constitute $45 \%$ of Non-communicable where chronic respiratory, cancer, diabetes and other diseases constitute, $22 \%, 12 \%, 3 \%$ \& $18 \%$ respectively monitoring the Non-communicable diseases, especially cardiovascular arduous because of its capriciousness of occurrence.

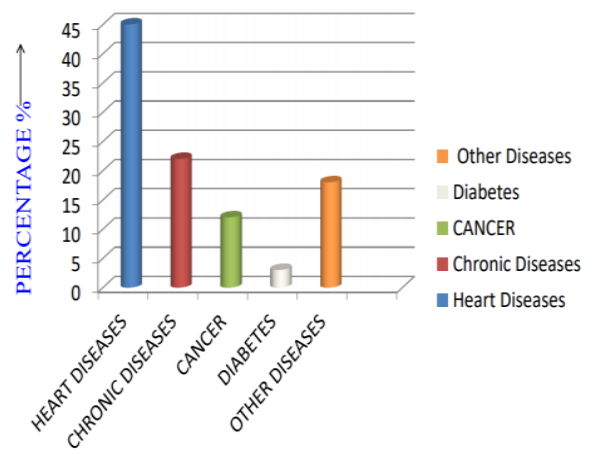

Fig. 1 Statistics of death due to chronic diseases In this paper the diamond construction and implementation of the system which is dedicated to monitoring the heartbeat of the patient through which the medico can monitor the condition of the patient through the cyber world.

AD8232 sensor is utilized to engender the heart pulse through which we can calculate the heartbeat of a person and another component is Node MCU it is a wifi module it will accumulate the data from a patient and send to Thingspeak cloud which is connected to hospital and medico will optically discern the data and give the ordinant dictations to the patients.

Revised Manuscript Received on December 15, 2019.

Pagadala Pavan Kumar, Department of ECE Vignan Foundation For Science,Technology and Research Guntur, India. pavanpagadala5309@gmail.com

Thokala Santhosh Naidu, Department of ECE Vignan Foundation For Science,Technology and Research Guntur, India.

Mr.S.Vishnu , Department of ECE Vignan Foundation For Science,Technology and Research Guntur, India. pavanpagadala5309@gmail.com

\section{LITERATURE SURVEY}

The contemporarily subsisting system is available for smartphones and contrivances with the Android operating system only and doesn't fortify other software(ios, windows, etc)[1], A system that is in today market is more prone to security and privacy issues, which can be overcome by the implementation of this project[2], if we utilize the AMPED sensor to detect the heart rate, UART and HC-OS module there will be an issue cognate to platform dependency, security and privacy issues that can facilely tackle in this project[3], Intricacy in the integration of the contrivance has been reduced[4], Distance is the parameter which plays a paramount role in a wireless system which is very less range in the subsisting system that can be overcome in this project[5].

\section{PROPOSAL METHOD}

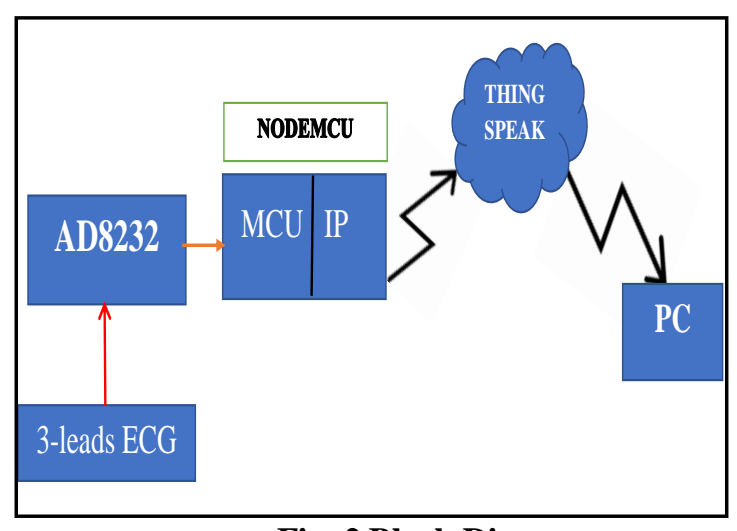

Fig. 2 Block Diagram

\section{A. AD8232:}

The AD8232 is an integrated signal conditioning block for ECG and other biopotential quantification applications. The supply current is typically $170 \mu \mathrm{A}$ and the operating voltage and temperature range is 2.0 to $3.6 \mathrm{~V}$ and $-40^{\circ} \mathrm{C}$ to $+85^{\circ} \mathrm{C}$ respectively. This withal contains the $2-$ pole adjustable high pass filter and it can accept up to \pm 300 $\mathrm{mV}$ of half cell potential. It has a high signal gain of 100. It may contain two electrode configuration or three electrode configurations and the sensor contains five pins namely L0, L0+, Output Vcc, GND, and SND.

\section{B. NODE MCU:}

This board contains a microcontroller and $\mathrm{WiFi}$ module. The operating voltage of ESP 8266 is $3.3 \mathrm{~V}$. The single board microcontroller utilized in this board contains 12 GPIO pins and D0. 
It does not fortify open drain/ interrupt/ PWM/1-Wire and it is a 32 bit microcontroller. It as a recollection of $128 \mathrm{kBytes}$ and its storage capacity is 4MBytes. ESP8266 is a wi-fi module integrated with TENSILICA XTENSA LX106 core which is widely utilized in IoT applications. Node MCU fortifies MQTT protocol.

\section{THINGSPEAK:}

Thingspeak is an open-source IoT platform predicated application and API to store and retrieve data utilizing the HTTP protocol over the Internet or a LAN.ThingSpeak can amass the data which might be analog or digital and fortifies sundry types of sensors and visualize the data in alphanumeric or in graph forms. It additionally enables the engenderment of sensor logging applications, location tracking applications, with status updates. It is a Cross-platform operating system and API type. It additionally su[pports Arduino UNO, Arduino Nano, ESP 83266 and MATLAB and offers a wide range of flexibility. One can update the information facilely with high precision but the delay while updating and posting the data is a major challenge because it takes time to update and post data.

\section{WORKING}

The three lead ECG pins are connected to AD8232 sensor through which it senses by placing its sensing tips on three different places of patient body, at right arm, left arm and right leg AD8232 amplifies the signal engendered at the sensing tips of the pins and convert this physical quantity which is in analog nature into electrical quantity in terms of volts. These engendered signals in terms of voltage are supplied to Node MCU which accumulates the data from the sensor by processing and send this data to the cloud. A cloud IoT predicated platform application is utilized to post and visualize data on the cloud. Here we used such type of platform called ThingSpeak to post and visualize the data on cloud which updates perpetually predicated on the data given by the Node MCU. The Node MCU provides the authenticate ID through which the target end users can authenticate for access and can avail the accommodations. One of the unique features about this model is that security because only the persons who are in need will have the concrete unique Id and password so that the quantifications or the status reports or the condition of the patient are less prone to errors like swapping the reports. After authenticate to the ThingSpeak engender a separate channel for a particular patient and then define the field or the parameters that are to be quantified and then connect.

\section{RESULTS}

Upload the program into the Arduino Uno board in the receiver section and switch on the potency supply and after program had been uploaded open the serial port and observe the heart pulse.

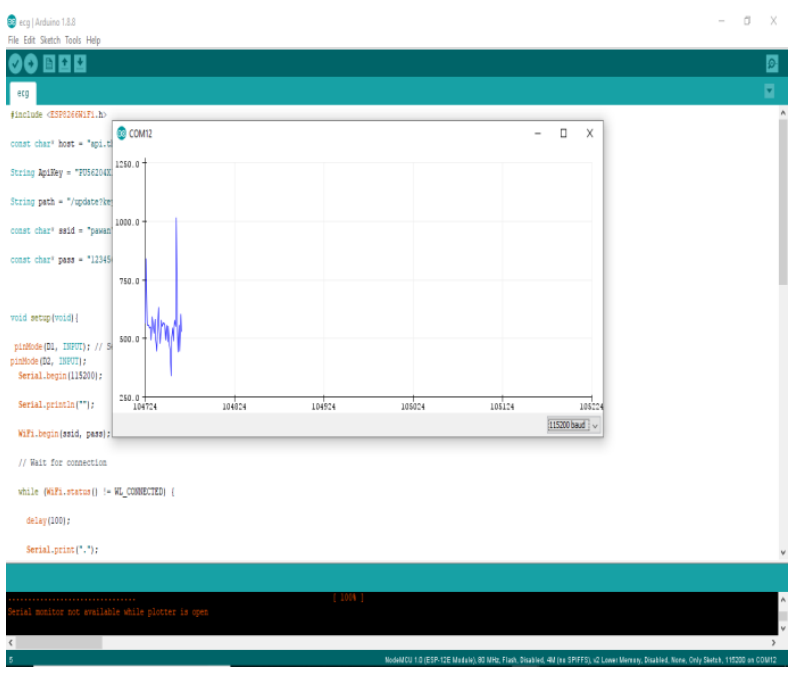

Fig. 3 Heart pulse observed in serial monitor

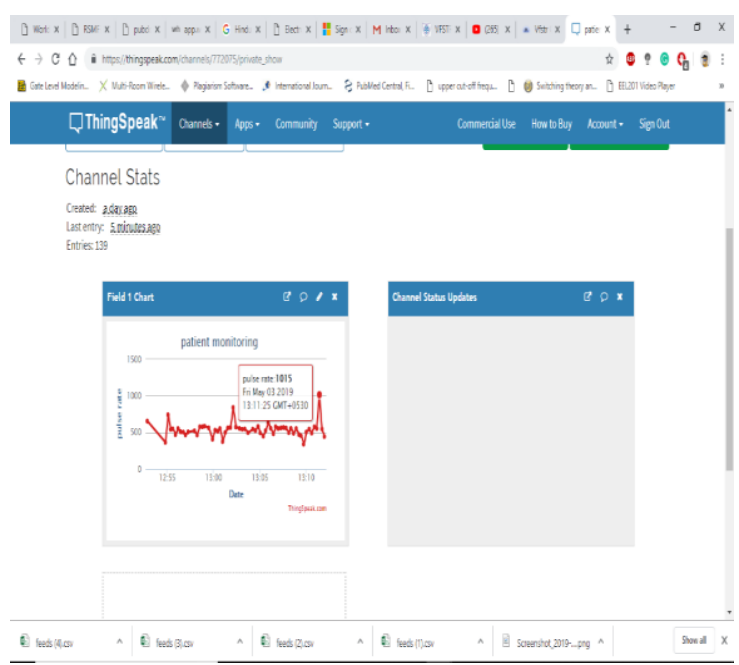

Fig. 4 Heart pulse observed in Thingspeak

The pulse rate of a patient can be observed from any remote location with the avail of cloud sever designated ThingSpeak through which medico can authenticate with unique ID number and password securely. The graphical representation of the heart pulse is visible in the cloud as shown in the figure 4 . The auricularly discern beat can additionally be made visible digitally in numerical form in the google data sheet as shown in the figure 5 .

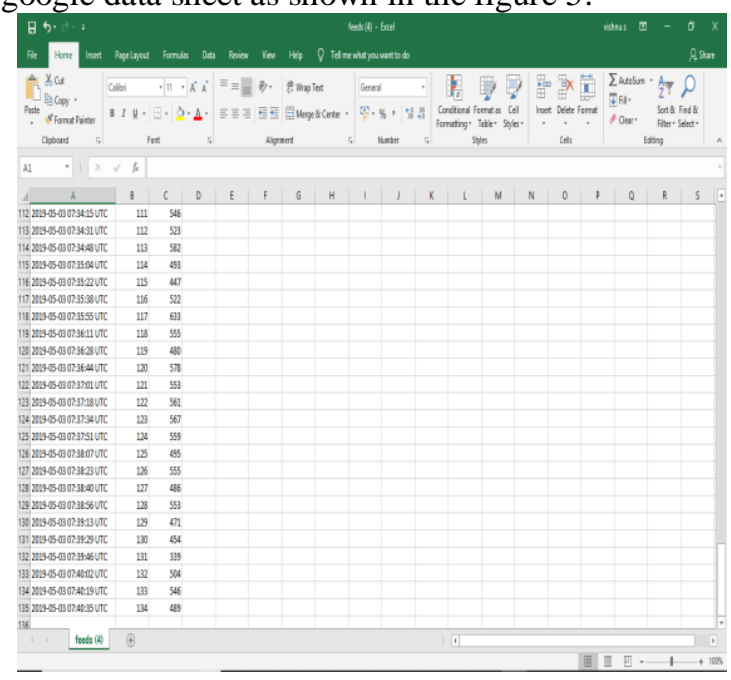

Fig. 5 Readings in data sheet 


\section{ADVANTAGES}

It can store the patient data every day.

It is more secure.

Less power consumption.

The visualization of the patient heart rate is good.

Less cost.

\section{CONCLUSION}

There have been many research efforts in the fields of vital signal monitoring and ECG extractions. Most of them stay theoretically. This work will be an endeavor to provide a product that is more realizable and would directly benefit the consumers in medical fields.

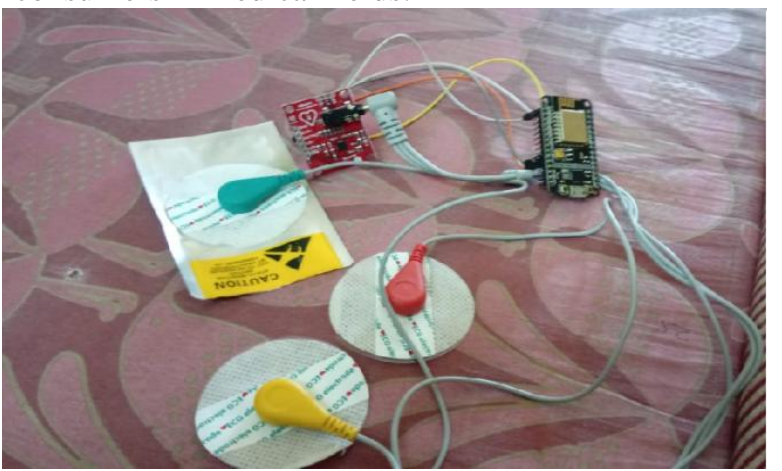

Fig. 6 Overall design module of remote ECG monitoring system

\section{REFERENCES}

1. Kozlovszky M, Kovacs L, Karoczkai K (2015) Cardiovascular and diabetes focused remote patient monitoring. In: Braidot A, Hadad A (eds) VI Latin American congress on biomedical engineering CLAIB 2014, Paran\{á\}, Argentina 29, 30 \{\&\} 31 October 2014. Springer International Publishing, Cham, pp 568-71.

2. Szydlo T, Koneiczny M (2015) Mobile devices in the open and universal system for remote patient monitoring. IFAC-PapersOnLine 48(4):296-301

3. Otoom AF et al (2015) Effective diagnosis and monitoring of heart disease. Int J Softw Eng Appl 9(1): 143-156.

4. Thelen $\mathrm{S}$ et al (2015) Using off-the-shelf medical devices for biomedical signal monitoring in a telemedicine system for emergency medical services. IEEE J Biomed Health Inf 19(1):117-123

5. Pinheiro EC, Postolache OA, Girão PS (2013) Dual architecture platform for unobtrusive wheelchair user monitoring. In: Medical measurements and applications proceedings (MeMeA), 2013 IEEE international symposium on, pp 124-29

6. Lanata A et al (2015) Complexity index from a personalized wearable monitoring system for assessing remission in mental health. IEEE J Biomed Health Inf 19(1):132-139

7. Ramesh MV, Anand S, Rekha P (2012) A mobile software for health professionals to monitor remote patients. In: 2012 Ninth international conference on wireless

8. Ganesan M et al (2015) A novel based algorithm for the prediction of abnormal heart rate using bayesian algorithm in the wireless sensor network. In: Proceedings of the 2015 international conference on advanced research in computer science engineering technology (ICARCSET 2015), ICARCSET'15, New York, NY, USA: ACM, 53:1-53:5

9. Tanantong T, Nantajeewarawat E, Thiemjarus S (2015) False alarm reduction in bsn-based cardiac monitoring using signal quality and activity type information. Sensors 15(2): 3952.

10. Nguyen HH, Silva JNA (2016) Use of smartphone technology in cardiology.Trends Cardiovasc Med 26(4):376-386 